\title{
Dienstleistungspolitik und Dienstleistungs- forschung - Ein Plädoyer für neue Impulse
}

Die Bedeutung von Dienstleistungen hat in den letzten Jahren zugenommen, aber eine Dienstleistungspolitik als eigenständiges Handlungsfeld ist kaum erkennbar. Die Förderung von Dienstleistungen erfolgte vornehmlich über Kostensenkungen, Liberalisierungen und Privatisierungen, die zulasten der Qualität der Arbeitsbedingungen und häufig auch der ServiceQualität gingen. Demgegenüber tritt die Dienstleistungsgewerkschaft ver.di für eine Dienstleistungspolitik ein, die für qualitativ hochwertige Dienstleistungen sorgt und die Prinzipien guter Arbeit in Dienstleistungsbranchen durchsetzt. Dienstleistungen sollen den gesellschaftlichen Strukturwandel gestalten und Ziele wie etwa den sozialen und ökologischen Umbau der Wirtschaft unterstützen.

Die herausragende und über die Jahre erheblich gewachsene ökonomische Relevanz des Dienstleistungssektors ist unstrittig: 29,4 Mio. Menschen waren in Deutschland im Jahr 2009 im Dienstleistungssektor beschäftigt, was einem Anteil von $73 \%$ der Erwerbstätigen entspricht. Der Beitrag des Dienstleistungssektors zur Wertschöpfung lag in diesem Zeitraum bei 72,6\%.' Die Bedeutung von Dienstleistungen für die gesamtwirtschaftliche Wertschöpfung und Beschäftigung hat dabei in den zurückliegenden Dekaden stark zugenommen: Die Anzahl der hier Erwerbstätigen ist zwischen 1970 (14,5 Mio.) und 2009 um mehr als das Doppelte gestiegen, der tertiäre Wertschöpfungsanteil lag 1970 noch bei 48,3\%. ${ }^{2}$ Am Megatrend der Tertiarisierung hat sich auch in der Wirtschafts- und Finanzkrise der letzten Jahre nichts geändert: Während in der Industrie in den Jahren 2009 und 2010 Zahlen des Statistischen Bundesamtes zufolge - hierzulande 366.000 Arbeitsplätze verloren gingen, entstanden im Dienstleistungssektor im gleichen Zeitraum 545.000 Jobs. $^{3}$
Deutschland ist - nimmt man diese Daten zum Maßstab - längst zu einer Dienstleistungsökonomie geworden. Dies festzustellen bedeutet nicht, die nach wie vor wichtige Rolle der industriellen Basis für Wachstum und Arbeitsplatzentwicklung zu relativieren oder gar zu negieren. Es gibt aber Anlass zu der Frage, ob die tektonische Verschiebung in den sektoralen Strukturen unserer Volkswirtschaft von Politik und Wissenschaft bereits hinreichend wahrgenommen und angemessen verarbeitet wurde. Hier sind erhebliche Zweifel angebracht und neue Impulse scheinen dringend vonnöten.

Zunächst ist $\mathrm{zu}$ konstatieren, dass Dienstleistungspolitik als eigenständiges und kohärentes politisches Handlungsfeld in der öffentlichen Wahrnehmung kaum präsent ist. Dies mag zum einen einer terminologischen Unschärfe geschuldet sein: Während „Industriepolitik“ zwar kontrovers diskutiert, aber doch in einem weitreichenden Konsens verstanden wird als das Ensemble politischer Interventionen zur Beeinflussung von Strukturen und Prozessen in der Güterproduktion und einzelner ihrer Branchen, so mangelt es dem - weit weniger gebräuchlichen - begrifflichen Pendant für den tertiären Bereich bislang an einem entsprechend gängigen Verständnis. Dabei geht es doch im Kern um ein und denselben Ansatz: Dienstleistungspolitik soll auf die Entwicklung dieses höchst heterogenen Sektors im Sinne bestimmter politischer Zielsetzungen einwirken - etwa um Beschäftigung zu schaffen, ökologische Innovationen voranzutreiben oder um in einem allgemeineren Sinn den Strukturwandel sozial zu flankieren. ${ }^{4}$ Dienstleistungspolitik will die Entwicklung der Dienstleistungen fördern. Sie muss dabei die Entwicklung der Nachfrage ebenso im Blick haben wie die des Dienstleistungsangebotes und - aus Sicht der Gewerkschaften von besonderer
Bedeutung - auch die Arbeitsbedingungen derjenigen, die Dienstleistungen erbringen.

Dass eine so verstandene Dienstleistungspolitik als eigenständiges, systematisch gedachtes und konkret gefasstes Handlungsfeld in Deutschland bestenfalls am Anfang steht, dürfte ein zweiter Grund für die unterentwickelte öffentliche Aufmerksamkeit sein, welche wiederum die politische Nichtbefassung im Wege einer Art Rückkopplungsschleife stabilisiert. Kritisch zu konstatieren bleibt in jedem Fall ein unzureichendes Verständnis und ein Hinterherhinken der Politik hinter dem rasanten Aufstieg des Dienstleistungsbereichs zum Leitsektor der deutschen Volkswirtschaft. Zugegeben: Gerade das schnelle Wachstum und auch die Unübersichtlichkeit der Dienstleistungsbranchen machen eine konsistente und strukturierte Dienstleistungspolitik schwierig. Worin liegt die Gemeinsamkeit von industrienahen Services, öffentlicher Verwaltung, dem Gesundheitswesen, den Medien, von Großhandel und Finanzdienstleistungen, haushaltsnahen Dienstleistungen und der Erbringung von Telekommunikationsdiensten, um nur wenige herauszugreifen? Sie unterscheiden sich in Wertschöpfung, Produktivität, in

\footnotetext{
1 Niebel, T. (2010): Der Dienstleistungssektor in Deutschland - Abgrenzung und empirische Evidenz, ZEW-Dokumentation 10-01, Mannheim, S. $3 \mathrm{ff}$.

2 Vgl. ebd.

3 Vgl. Kaelble, M./Ohanian, M./Rademaker, M. (2011): Industriewunder entpuppt sich als Märchen, in: Financial Times Deutschland vom 04.01.

4 Legte man das weiter gefasste, eben nicht nur auf den sekundären Sektor bezogene angloamerikanische Verständnis von "industry“ bzw. "industrial policy“ zugrunde, so wäre „Dienstleistungspolitik“ im Kern nichts anderes als "industrial policy" für den Dienstleistungssektor.
}

Frank Bsirske ist Vorsitzender der Vereinten Dienstleistungsgewerkschaft (ver.di). 
der Qualität der Arbeit und den Arbeitsbedingungen, auch im gesellschaftlichen Ansehen. Dessen ungeachtet kann es sich Politik angesichts der eminenten ökonomischen und gesellschaftlichen Bedeutung des Dienstleistungssektors nicht erlauben, vor dieser Heterogenität zu kapitulieren und weiterhin auf gestaltende Intervention im Sinne positiver, konsistenter Strategieentwürfe zu verzichten.

\section{DEREGULIERUNG UND KOSTEN- SENKUNG ALS WEG ZU MEHR DIENSTLEISTUNGEN?}

Obwohl von einer Dienstleistungspolitik im eigentlichen Wortsinn bislang also kaum die Rede sein kann, so waren in den zurückliegenden Jahren gleichwohl politische Versatzstücke einer solchen zu verzeichnen, und sie haben die Entwicklung des Sektors - zumal seiner Beschäftigungsverhältnisse, Arbeits- und Einkommensbedingungen - erheblich geprägt. Sie bestanden überwiegend in voneinander isolierten, zumeist beschäftigungspolitisch verpackten Ansätzen zur Kostensenkung im einseitigen Interesse der Wirtschaft. Ihr Ausgangspunkt war die angebliche Dienstleistungslücke in Deutschland (u.a. gemessen an den USA), die Mitte der 1990er Jahre von Wirtschaftsinstituten, Arbeitgeberverbänden und Medien „entdeckt" worden war. Um diese zu schließen, verfolgte eine zunehmend neoliberal geprägte Politik vorrangig das Ziel, das Dienstleistungsangebot durch Liberalisierung, Privatisierung und Deregulierung $\mathrm{zu}$ erweitern und mittels einer gezielten „Low-road-Strategie“ zur Reduzierung von Transaktions- wie Arbeitskosten zu verbilligen. Indem der öffentliche Sektor zurückgeschnitten und für privates Kapital geöffnet wurde, sollten Investoren angelockt und durch Deregulierung des Arbeitsmarktes Beschäftigungsmöglichkeiten geschaffen werden. Die Förderung von Dienstleistungen - im quantitativen, nicht im qualitativen Sinne - durch die Umwandlung öffentlicher in private Angebote und die Verbilligung der Ware Arbeitskraft in einer Vielzahl von Segmenten des Dienstleistungssektors kennzeichnen seitdem den Mainstream einer Politik unter der Devise: Billiger statt besser.

Im Ergebnis dieser Politik sank das Angebot an öffentlichen Dienstleistungen, während die gesellschaftlichen Problemlagen zunahmen und mehr und mehr gravierende Defizite im Bildungswesen, im Gesundheitswesen, im Umweltschutz und der öffentlichen Infrastruktur zutage traten. Die "Ausblutungsstrategie ${ }^{{ }_{5}}$ gegenüber dem öffentlichen Dienst hat die in sie gesetzten Hoffnungen nicht erfüllt. Keineswegs stieg die Servicequalität im versprochenen Maße. Im Gegenteil: Personalabbau und die Verschlechterung der Arbeitsbedingungen wirken qualitätssenkend, und auch die Kosten für die Nutzer und Verbraucher stiegen in vielen Bereichen. Gleichzeitig konnte die zahlungsfähige private Nachfrage nach bestimmten Dienstleistungen - etwa in der Pflege - aufgrund der schwachen Massenkaufkraft nicht mit dem objektiv steigenden Bedarf Schritt halten. Auf diese Weise wurde ein Entwicklungspfad für den Dienstleistungssektor zementiert, der primär auf Kostenkonkurrenz und kaum auf Innovationen, Investitionen und den systematischen Ausbau von qualitativ hochwertigen Services setzt.

Schon zur Zeit der Regierung Kohl sollten die Arbeitskosten durch Sozialabbau wie durch Schritte zur Deregulierung des Arbeitsmarktes gesenkt werden. Unter der Devise, Beschäftigungshemmnisse zu beseitigen, wurden im Jahre 1996 die sachgrundlose Befristung zugelassen, der Kündigungsschutz gelockert, die Sozialabgaben auf unter $40 \%$ gedrückt. Eine Senkung der Lohnfortzahlung auf $80 \%$ scheiterte weitgehend am Widerstand der Gewerkschaften. Gleichzeitig verlangten die Arbeitgeber niedrigere Einstiegstarife für Angelernte und Geringqualifizierte, förderten die Auflösung der Tarifbindung durch Verbandsaustritt sowie durch betriebliche Öffnungsklauseln, beharrten auf Lohndisparitäten zwischen Ost und West und setzten den bisherigen Mechanismus zur Allgemeinverbindlicherklärung von Tarifverträgen vor allem im Dienstleistungssektor außer Kraft. Zugleich ergingen sich Arbeitgeberfunktionäre, Wirtschaftswissenschaftler und Freizeitforscher in Visionen von Heerscharen neuer Dienstleistungsarbeiter: als Hilfspacker im Supermarkt, für Dienstbotentätigkeit im Haushalt, als Schuhputzer im Bahnhof usw.

Die rot-grüne Bundesregierung hingegen sorgte in ihrer ersten Regierungszeit für die Wiederherstellung der hundertprozentigen Lohnfortzahlung im Krankheitsfall und des Kündigungsschutzes sowie für die Regulierung geringfügiger Beschäftigung, der 630-DM-Jobs, und die
Bekämpfung der Scheinselbstständigkeit. Unter dem Eindruck steigender Arbeitslosigkeit und wachsender Defizite in den Sozialkassen vollzog dann die Regierung Schröder im Jahre 2003 mit der Agenda 2010 jedoch einen Paradigmenwechsel in der Arbeitsmarktpolitik mit dem erklärten Ziel, die Arbeitslosigkeit durch Druck auf Arbeitslose, Leistungskürzungen und Sanktionen auf der einen und den Ausbau des Niedriglohnsektors auf der anderen Seite zu überwinden. Die Maßnahmen sind bekannt: Zusammenfassung von Arbeitslosen- und Sozialhilfe auf dem Niveau der Sozialhilfe, Verschärfung der Zumutbarkeit und der Sanktionen auf der einen Seite, auf der anderen die Unterstützung von Soloselbstständigkeit per Ich-AG, der Ausbau der 400-Euro-Jobs durch Abschaffung der zeitlichen Begrenzung, die Deregulierung der Leiharbeit und die Förderung von Ein-Euro-Jobs, mit dem Ziel, die Arbeitsfähigkeit Arbeitsuchender zu testen, und dem Effekt, einen erheblichen Teil regulärer Arbeit zu ersetzen.

Bekannt ist auch das Ergebnis dieser Politik: Bereits im Aufschwung 2005 bis 2008 stieg das Arbeitsvolumen, während die Reallöhne im Durchschnitt sanken. Die neue Beschäftigung führte zum Ausbau des Niedriglohnsektors, zum Anstieg der Leiharbeit, zur Ausweitung der Minijobs auf über 5 Mio., zur Zunahme der Soloselbstständigen und insgesamt zu einer Expansion des Niedriglohnsektors auf eine Größenordnung von fast $20 \%$ aller Erwerbstätigen. Die Strategie, Dienstleistungsbeschäftigung durch Lohndumping und Entsicherung der Arbeitsverhältnisse quantitativ zu fördern, sorgte auch für eine Erosion der Arbeitsbedingungen in der Industrie. Dienstleistungen, die in Industrieunternehmen bislang auch zu Tarifen der Industriearbeit erbracht worden sind, wurden mehr und mehr ausgegliedert. Standen anfangs die Kantinen, der Wachdienst, Transport und Logistik oder die Instandsetzung auf dem Plan, so sind es heute die Finanzdienstleistungen und große Teile der Forschung und Entwicklung (FuE). Zudem hielt in der Industrie Leiharbeit Einzug. Anfangs, um Produktionsspitzen und krankheits- oder urlaubsbedingte Ausfälle auszugleichen, mit der Zeit mehr

\footnotetext{
5 Vgl. hierzu Heintze, C. (2010): Die Entfaltung sozi aler Dienstleistungen, in: Gegenblende (01), www. gegenblende.de/01-2010/++co++a9007774 e347-11de-43a9-00093d10fae2.
} 
und mehr, um vor allem in der Automobilzulieferindustrie und der chemischen Industrie Stammbelegschaften zu ersetzen und Arbeitskosten zu senken.

Bis heute - und auch über die Weltwirtschaftskrise hinweg - hat diese Politik ihre Fortsetzung gefunden: Einnahmeausfälle der öffentlichen Hand, verursacht durch Steuersenkungen und die Wirkungen der Krise, erhöhen den Druck zum Abbau öffentlicher Angebote. Eine neue Welle der Liberalisierung von Dienstleistungen wird auf europäischer Ebene vorbereitet. Die einseitig auf den Export orientierte Politik vernachlässigt nach wie vor die Binnennachfrage. Gleichzeitig wächst mit dem Arbeitsvolumen die Zahl der prekären Arbeitsverhältnisse: Fast 7 Mio. Minijobs, über 4,2 Mio. Teilzeitbeschäftigte, ein rasanter Anstieg der befristeten Beschäftigungsverhältnisse - nur noch $38 \%$ aller ausgebildeten jungen Erwachsenen finden eine unbefristete Anstellung - und fast eine Million Beschäftigte in Leiharbeit, davon mehr als Zweidrittel in einer Beschäftigung von weniger als sechs Monaten. Zugleich gerät der Verzicht auf eine zukunftsorientierte, auf Qualität, Nachhaltigkeit und Innovation gerichtete Dienstleistungspolitik zum gesellschaftlichen Skandal: Wachsende Bildungsarmut, Pflegenotstand, eine vernachlässigte öffentliche Infrastruktur, Defizite im Umweltschutz und eine öffentliche Verwaltung, die den neuen und schwierigen Herausforderungen, wie sie sich etwa mit sozialer Ausgrenzung und zunehmender Interkulturalität verbinden, kaum mehr gerecht werden kann.

In der Gesamtschau ist in Übereinstimmung mit Cornelia Heintze zu konstatieren: „Sieht man von den unternehmensnahen Dienstleistungen ab, weist Deutschlands Weg in die Dienstleistungsgesellschaft Anzeichen einer deformierten Entwicklung auf. Bei sozialen und gesellschaftsnahen Dienstleistungen gilt dies im Besonderen. [...] Deutschland fällt nicht nur gegenüber den fünf nordisch-skandinavischen Ländern mit ihren weltweit am höchsten entwickelten Wohlfahrtsstaaten immer weiter zurück, sondern hat auch gegenüber anderen Ländern an Terrain eingebüßt."

\section{ZIELE UND FELDER EINER DIENST- LEISTUNGSPOLITIK, DIE IHREN NAMEN VERDIENT}

In der öffentlichen Debatte wie auch in der Meinungsbildung von Politik, Verbänden und gesellschaftlichen Gruppen wächst die Einsicht, dass es so nicht weitergehen kann: Weder für einen Börsengang der Bahn noch für eine Privatisierung des Öffentlichen Personennahverkehrs (ÖPNV) finden sich Mehrheiten, Rekommunalisierung steht auf der Tagesordnung vieler Städte, Investitionen und mehr Chancengleichheit im Bildungswesen rangieren in der politischen Programmatik weit oben, die Zustände in Krankenhäusern und der Altenpflege werden als untragbar empfunden, und die Forderung nach einem gesetzlichen Mindestlohn und einer Reregulierung der Leiharbeit erfährt breite öffentliche Unterstützung. Generell ist im politischen Spektrum links von FDP und neoliberalem Flügel der Union der Ruf nach Korrekturen und Reformen deutlich zu vernehmen.

Eine Dienstleistungspolitik müsste strategisch angelegt sein, einen ganzheitlichen Ansatz verfolgen und so zugespitzt werden, dass sie nicht nur öffentliche Aufmerksamkeit und Unterstützung findet, sondern auch politisch wirksam wird. Ziel einer solchen Politik wäre es,

- die Nachfrage nach qualitativ hochwertigen öffentlichen wie privaten Dienstleistungen zu steigern;

- das Angebot vor allem öffentlicher Dienstleistungen an gesellschaftlichen Bedarfen auszurichten und allen, unabhängig vom Einkommen, einen gleichen Zugang zu ihnen zu gewährleisten;

- Dienstleistungen an den Bedürfnissen der Menschen, die sie in Anspruch nehmen, ebenso auszurichten wie am Erfordernis einer guten Arbeit für diejenigen, die sie erbringen;

- somit die Qualität der Dienstleistungen wie die der Dienstleistungsarbeit durch Innovationen zu verbessern und zu diesem Zweck Dienstleistungsforschung und -entwicklung auszubauen;

- die Bedeutung der Dienstleistungen für Wachstum und Beschäftigung im öffentlichen Bewusstsein hervorzuheben;

- Dienstleistungsarbeit in doppeltem Sinne aufzuwerten: bzgl. der gesellschaftlichen Anerkennung wie im Entgelt und der Gestaltung der Arbeitsbedingungen.

Unter diesen gemeinsamen Zielsetzungen vereinigen sich verschiedene Handlungsebenen, die sowohl der Differenziertheit der Ziele wie der Heterogenität des Dienstleistungssektors geschuldet sind.
Wirtschaftspolitisch steht der Abbau der für die deutsche Wirtschaft typischen Ungleichgewichte zwischen Export und Binnennachfrage im Vordergrund. Höhere Löhne, eine gerechtere Verteilung der Arbeitszeit zwischen den Geschlechtern und eine stärkere öffentliche wie private Nachfrage, finanziert über eine Steuerpolitik, die an Stelle der Umverteilung von unten nach oben auf die Stärkung der unteren Einkommensschichten setzt. Vermögenssteuer, höhere Erbschaftsteuer, ein höherer Spitzensteuersatz, eine Finanztransaktionssteuer und eine Gemeindewirtschaftssteuer, um nur die wichtigsten zu nennen, sind geeignete Instrumente für einen wieder handlungsfähigeren Staat.

Ein solcher ist unabdingbare Voraussetzung, um die öffentlichen Dienstleistungen nach skandinavischem Vorbild auszubauen und die überfälligen Investitionen in Infrastruktur und Daseinsvorsorge zu finanzieren: Allein der jährliche Investitionsbedarf für die Bildung - von der Kindertagesstätte bis zur Hochschule - wird von Experten auf bis zu $36 \mathrm{Mrd}$. beziffert, als Investitionen in Gebäude wie in Köpfe. Über 50 Mrd. aus den Kassen der Länder wären nötig, um den Investitionsstau bei den Krankenhäusern aufzulösen, gleiches gilt für die Modernisierung der Pflegeheime. Eine der effektivsten Volkswirtschaften und der reichsten Gesellschaften der Welt hat über Jahre hinweg aufgrund einer verfehlten neoliberalen Politik den öffentlichen Sektor und damit wichtige gesellschaftliche Aufgaben vernachlässigt. Hier sind neue Weichenstellungen überfällig.

Öffentliche Investitionen in Milliardenhöhe erleichtern die Lösung von Struktur-und Handlungsdefiziten in der öffentlichen Daseinsvorsorge. Sie verbessern das Angebot im Bildungswesen, im Gesundheitswesen, im Umweltschutz, in der Entwicklung der Städte und sichern somit die gleichberechtigte Teilhabe aller, unabhängig vom Einkommen. Gerade in den letzten Jahren zogen die wachsenden Defizite im öffentlichen Dienstleistungstableau den Ausbau privater Angebote nach sich - von der privaten Kindertagesstätte über die Privatschule, die private Hochschule und die Privatklinik bis zum privaten Pflegeheim -, zugleich boomen die nur Wohlhabenden zugänglichen Freizeit-

6 Ebd., S. 1. 
einrichtungen, ja selbst Sicherheit ist längst kein allein öffentlich erstelltes Gut mehr. Dass sich nur Reiche einen armen Staat leisten können, darauf hat schon Willy Brandt vor mehr als 30 Jahren hingewiesen.

So unabweisbar die Handlungsbedarfe zur Ausweitung und Optimierung öffentlicher Dienstleistungen sind, so dringlich - und differenzierter als bislang - gilt es, die Frage nach dem Spannungsverhältnis zwischen den Bedürfnissen derer, die Dienstleistungen in Anspruch nehmen, und den Interessen derer, die sie erbringen, zu thematisieren. So lassen sich zum Beispiel der Wunsch von Eltern nach einer ihren Arbeitszeiten angemessenen Kinderbetreuung und das Interesse der Erzieherinnen und Erzieher an klar geregelten Arbeitszeiten nicht einfach in Übereinstimmung bringen. Gleiches gilt für die Servicezeiten von Behörden, den ÖPNV, den Bereitschaftsdienst in Krankenhäusern. Arbeits- und Lebenszeiten richten sich immer weniger an festen Zeitstrukturen aus. Die Konflikte und Übereinstimmungen in den Zeitkontingenten und -bedarfen von Nutzern und Erbringern von Dienstleistungen, von Eltern, Kunden, Patienten, Arbeitsuchenden usw. sind ein wichtiger Aspekt einer notwendigen gesellschaftlichen Zukunftsdebatte.

Derlei „Zeitfragen“ berühren ein weiteres, aus gewerkschaftlicher Sicht zentral bedeutsames Handlungsfeld von Dienstleistungspolitik: Die Verbesserung der Arbeitsqualität der Beschäftigten, die Realisierung von guter Arbeit als notwendiger Voraussetzung guter Dienstleistungen. Hier steht aktuell die Auseinandersetzung mit der zunehmenden Prekarisierung der Arbeit im tertiären Sektor im Vordergrund. Die zunehmende Entsicherung in Verbindung mit sich ausbreitenden Niedriglöhnen widerspricht nicht nur den Wünschen der Beschäftigten nach guter Arbeit, sondern auch dem Bedarf von Kunden und Nutzern an guten Dienstleistungen. Dienstleistungsarbeit ist nämlich in weiten Teilen interaktive Arbeit, bei der die Erbringer unmittelbar mit den Nachfragern „umgehen“ müssen.

Soll dies in einer Weise vonstatten gehen, die für alle Beteiligten von maximalem Nutzen ist, so bedarf es einer gezielten Qualifizierung und forcierten Professionalisierung von Dienstleistungsarbeit. Dies beinhaltet sowohl eine verstärkte akademische Ausbildung als auch die Förderung von Beruflichkeit durch Ausbildung jenseits der Hochschule. Ausbildung darf nicht zu stark spezialisieren, sondern sie sollte in breit angelegten Kernberufen erfolgen. Es müssen neben den berufsspezifischen Fachkompetenzen vermehrt auch berufsübergreifende, erwerbsarbeitsbezogene Kompetenzen wie z.B. Kommunikationsfähigkeit und berufsunabhängige allgemeine kognitive Kompetenzen vermittelt werden. Letzeres zielt auch darauf ab, dass die Beschäftigten in die Lage versetzt werden, die Konsequenzen von Veränderungen in der Arbeitswelt zu reflektieren und etwa individuellen Weiterbildungsbedarf reklamieren können. Dafür müssen aber auch die Möglichkeiten zur Weiterbildung durch gesetzliche, tarifliche und betriebliche Regelungen verbessert werden.

Eine weitere große Herausforderung, von der viele Dienstleistungsbranchen betroffen sind, ist die zunehmende Rationalisierung: Arbeit wird durch Technisierung komplett oder zum Teil auf Maschinen verlagert, über Veränderungen in der Arbeitsorganisation werden Abläufe verdichtet, zentralisiert und flexibilisiert, oder es wird ein Teil der Arbeit externalisiert und auf die Kunden verlagert. Häufig greifen diese Prozesse ineinander; den neuen technischen Möglichkeiten kommt dabei meist eine Treiberrolle zu. Der Trend eines rationalisierungsorientierten Einsatzes von Informations- und Kommunikationstechnologien (IKT) und Automatisierungstechniken kommt branchenübergreifend zum Tragen und bringt umfassende Veränderungen mit sich: Arbeits- und Herstellungsprozesse sowie Vertriebswege werden durch Automatisierung und IKT umgestaltet, neue Dienstleistungen entstehen ebenso wie neue Distributions- und Wertschöpfungswege. Im Zeichen von Kostensenkungsstrategien führen Digitalisierung und Automatisierung zu Personalabbau und setzen die verbliebenen Beschäftigten unter einen vermehrten Leistungsdruck. Arbeitsabläufe, z.B. im Bankensektor, werden nach industriellem Vorbild zerstückelt und mit wenig Rücksicht auf Fachwissen und ergonomische Anforderungen wieder zusammengesetzt. Der Leistungsdruck nimmt zu, und viele Beschäftigte fühlen sich durch den Technikeinsatz fremdbestimmt und überwacht.

Prinzipiell aber bietet die technologische Entwicklung aus Sicht der Beschäftigten auch Chancen. Mit ihr entstehen neue Produkte und Geschäftsmodelle, die die Grundlage für den Aufbau von Beschäf- tigung liefern können. Auch führt der Einsatz von Technik nicht zwangsläufig zur vollständigen Substitution von Arbeitskraft, sondern kann im Idealfall Arbeitserleichterungen schaffen. Im Bereich personenbezogener Dienstleistungen, etwa in einem Krankenhaus oder einer Pflegeeinrichtung, kann Technikeinsatz, etwa für Transport oder Überwachungstätigkeiten, den zeitlichen Spielraum für die eigentliche Pflegearbeit vergrößern und damit Beschäftigten- und Patienteninteressen dienen. Die Ausschöpfung dieser Chancen verlangt jedoch eine proaktive Gestaltung von Dienstleistungsarbeit und Dienstleistungsinnovationen unter intensiver Beteiligung der Interessenvertretungen. Gewerkschaften müssen auch in Dienstleistungsbereichen einen Technikeinsatz einfordern, der sich nicht einseitig an unternehmerischen Rationalisierungsund Kostensenkungsinteressen orientiert, sondern die Interessen von Beschäftigten und Nutzern von Beginn an integriert.

Leitbild ist ein Verständnis von Arbeit, das selbstbestimmtes Handeln ermöglicht, statt sich einseitig technischen Imperativen zu unterwerfen. Dies bedeutet auch, Innovationen und Innovationspolitik nicht auf rein technologische Entwicklungen zu reduzieren sowie Innovationsförderung nicht primär unter dem Gesichtspunkt einzelwirtschaftlicher Effizienzsteigerung zu betreiben. Aus gewerkschaftlicher Sicht sollen vielmehr die Qualität der Arbeitsverhältnisse und der Nutzen für die Verbraucher die entscheidenden Parameter für erstrebenswerte technologische Neuerungen und Dienstleistungsinnovationen sein.

\section{ANFORDERUNGEN AN DIE DIENST- LEISTUNGSFORSCHUNG}

Damit die Gewerkschaften und die Interessenvertretungen der Beschäftigten den technologischen oder arbeitsorganisatorischen Konzepten der Arbeitgeberseite Alternativen entgegensetzen können, müssen sie die wesentlichen Trends und Herausforderungen im tertiären Sektor rechtzeitig zur Kenntnis nehmen und analysieren. Aus dieser Notwendigkeit entspringt das ausgeprägte gewerkschaftliche Interesse an einer exzellent ausgestatteten und an zentralen gesellschaftlichen Problemlagen ausgerichteten Dienstleistungsforschung. Diese muss in Deutschland ausgeweitet und intensiviert werden, um der immens 
gestiegenen volkswirtschaftlichen Bedeutung von Dienstleistungen adäquat Rechnung tragen und neue, dringend benötigte Impulse für mehr Beschäftigung, gute Arbeit und nachhaltige Innovation geben zu können.

Dazu bedarf es nicht zuletzt einer substanziellen Erhöhung öffentlicher Fördermittel: In Relation zur mittlerweile deutlichen Dominanz und den nach wie vor erheblichen zusätzlichen Wertschöpfungs- und Beschäftigungspotenzialen tertiärer Ökonomie ${ }^{7}$ sowie im internationalen Vergleich fallen die auf Dienstleistungen gerichteten Anstrengungen bei Forschung und Entwicklung in Deutschland nämlich bei Weitem zu niedrig aus. In den OECDStaaten, in denen auf den Dienstleistungsbereich im Durchschnitt $22 \%$ der gesamten FuE-Ausgaben entfallen, kommt Deutschland mit einem Dienstleistungsforschungsanteil von lediglich $10 \%$ auf einen der hintersten Ränge. ${ }^{8}$ Dass sich der gesamtwirtschaftliche Strukturwandel hierzulande bis heute nicht in einer adäquaten Schwerpunktverlagerung bei den Forschungsaktivitäten niederschlägt, ist in erster Linie einer staatlichen FuEPolitik anzulasten, welche - ungeachtet vielfältiger gegenteiliger „Bekenntnisse“ zur Bedeutung des Dienstleistungssektors - erkennbar noch immer stark ,industrialistisch “ geprägt ist. Ein Indiz dafür ist die nach wie vor eklatante Unterfinanzierung der öffentlichen Förderprogramme für die Dienstleistungsforschung. ${ }^{9}$ So stehen beispielsweise für das 2006 gestartete Programm des Bundesministeriums für Bildung und Forschung (BMBF) „Innovationen mit Dienstleistungen“ für eine fünfjährige Laufzeit nur 70 Mio. $€$ an öffentlichen Mitteln zur Verfügung, während die "Hightech-Strategie" der Bundesregierung, deren Bestandteil das Förderprogramm ist, im gleichen Zeitraum mit insgesamt rund $15 \mathrm{Mrd}$. $€$ ausgestattet ist. $^{10}$

Die Forderung der Gewerkschaft ver.di nach einer deutlich höheren Dotierung und einem Ausbau der Dienstleistungsforschung antwortet auf drei Herausforderungen:

- erstens, auf den steigenden und sich zugleich ändernden Bedarf an Dienstleistungen für die Allgemeinheit, wie sie der demografische Wandel, die Veränderung von Lebensformen und Lebensstilen, die Gleichstellung der Geschlechter, die zu- nehmende Interkulturalität der Gesellschaft vor allem in den großen Städten, aber auch die Europäisierung und Globalisierung des öffentlichen Sektors von Wirtschaft und Gesellschaft verlangen; - zweitens, auf die wachsende Durchdringung von Dienstleistungen und Dienstleistungsarbeit mit Wissen und die erhöhten Anforderungen an Kreativität, soziale und kommunikative Kompetenz. Kunst, Kultur, Theater, Bildung, Design, Mode, Sport, Freizeit, Wellness sind keine Restgrößen, sondern wesentliche Treiber der Dienstleistungsentwicklung;

- drittens, auf die zunehmende Verflechtung von Industrie und Dienstleistungen in hybriden Wertschöpfungsketten, in denen ohne Services keine hochwertige industrielle Produktion und ohne diese keine wertschöpfenden Dienstleistungen möglich sind.

Aus gewerkschaftlicher Sicht erscheint jedoch nicht nur die Ausweitung, sondern auch eine Neuausrichtung der Dienstleistungsforschung vonnöten. Diese sollte stärker als bisher arbeitsorientierte Forschung sein und die Klärung der realen Bedingungen von Dienstleistungsarbeit sowie der Gestaltungsoptionen für "gute Dienstleistungsarbeit“ zu ihrem zentralen Gegenstand machen. Zwar berührt Dienstleistungsforschung auch bisher angesichts der engen Verschränkung von Produktions- und Arbeitsprozessen bei der Erbringung von Dienstleistungen naturgemäß immer wieder arbeits- und beschäftigungsorientierte Themen. Allerdings stellt sie dabei in aller Regel nicht „die Arbeits- und Beschäftigtenperspektive ins Zentrum" "11 ihrer Fragestellungen. Stattdessen ist überwiegend ,eine Fokussierung auf die Perspektive, die Interessen und die Handlungsmöglichkeiten des Einzelunternehmens festzustellen, “12 in welcher der Zielsetzung guter Dienstleistungsarbeit ein allenfalls instrumenteller Stellenwert zukommt: Eine höhere Arbeitsqualität ist in dieser Hinsicht dann und deshalb erstrebenswert, weil sie eine Steigerung von Motivation und Kreativität der Beschäftigten bewirkt, zur Generierung und Beschleunigung von Innovation beiträgt, zur Erhöhung von Kundenfreundlichkeit und Kundennutzen führen kann und damit letztlich für mehr Produktivität und Profitabilität zu sorgen vermag.

Zwar sind auf der Basis entsprechender Win-Win-Annahmen in Forschungs- und
Gestaltungsprojekten unter günstigen Rahmenbedingungen durchaus Interessenkonstellationen möglich, die sich sowohl für die Unternehmen wie auch für die Beschäftigten ertragreich machen lassen - und fraglos haben sich aus solchen Projekten auch immer wieder reale Verbesserungen der Arbeitsqualität ergeben. Geht man allerdings davon aus, dass der Dreh- und Angelpunkt einer gewerkschaftlich inspirierten Dienstleistungspolitik die Förderung guter Dienstleistungsarbeit sein muss, so kann ein vorrangig betriebswirtschaftlichen Postulaten verpflichteter Ansatz gleichwohl nur von begrenztem Nutzwert sein. Zum einen lassen sich unter solchen Prämissen Fragestellungen und Gestaltungsziele, welche „nur“ der Verbesserung der Arbeitsqualität dienen, ohne eine wenigstens vermittelte oder längerfristige produktivitäts- und profitabilitätssteigernde Wirkung versprechen zu können, kaum schlüssig begründen und deshalb bestenfalls auf einer Art „hidden agenda“ platzieren. Zum anderen haben sich die objektiven Voraussetzungen für derlei Win-Win-Arrangements in den zurückliegenden Jahren alles andere als verbessert, weil in zunehmend größer gewordenen Teilen des Managements die vormalige „Überzeugung, dass die subjektive Leistungsbereitschaft - insbesondere Kreativität und Innovation - von einem „pfleglichen Umgang“ der Unternehmen mit den Beschäftigten abhängig ist, [...] zur Disposition"13 steht. Stattdessen haben vielfach Orientierungen Platz gegriffen, welche „darauf basieren, den Beschäftig-

7 Vgl. Rheinisch-Westfälisches Institut für Wirtschaftsforschung (RWI) (2008): Potenziale des Dienstleistungssektors für Wachstum von Bruttowertschöpfung und Beschäftigung, Essen.

8 Daten von 2005 nach Legler, H./Krawczyk, O. (2009): FuE-Aktivitäten von Wirtschaft und Staat im internationalen Vergleich, Hannover, S. 75.

9 Vgl. hierzu Fraunhofer Institut für Arbeitswirtschaft und Organisation (IAO) (2008): Dienstleistungswirtschaft und Dienstleistungsforschung in Deutschland: Entwicklungen und Herausforderungen, Stuttgart, S. $24 \mathrm{ff}$.

10 Ebd., S. 28.

11 Sauer, D. (2003): Arbeit, Leistung und Interessenhandeln in der "tertiären" Organisation; in: Pohlmann, M./Sauer, D./Trautwein-Kalms, G. (Hrsg.): Dienstleistungsarbeit: Auf dem Boden der Tatsachen - Befunde aus Handel, Industrie, Medien und IT-Branche, Berlin, S. 15-25 (hier: S. 15).

12 Social Science Service Research (2010): Positionspapier der Initiative "Social Science Service Research" (3SR), o.O., S. 11.

13 Marrs, K. (2008): Arbeit unter Marktdruck. Die Logik der ökonomischen Steuerung in der Dienstleistungsarbeit, Berlin, S. 24. 
ten zu vermitteln, dass sie selbst zu einer austauschbaren "Commodity" geworden sind. " ${ }^{14}$

Vor diesem Hintergrund benötigt Dienstleistungsforschung eine erweiterte Perspektive, in welcher Arbeit nicht nur als Ressource und gute Dienstleistungsarbeit nicht nur als instrumenteller „Enabler“ erhöhter Produktivität und Profitabilität von Unternehmen erscheint, sondern als eigenständiges, mit betriebswirtschaftlichen Zielsetzungen zumindest gleichrangiges Forschungs- und Gestaltungsziel. Eine solche Aufwertung arbeitsorientierter Dienstleistungsforschung setzt eine veränderte Konzipierung von Förderschwerpunkten und Ausschreibungstexten ebenso voraus wie eine intensivere gewerkschaftliche Beteiligung an den Entscheidungsprozessen der Forschungsförderung und eine erweiterte Einflussnahme der Beschäftigten und ihrer Interessenvertretungen bei der Initiierung und Durchführung von Projekten. Ver.di kann und will so verstandene "Dienstleistungsarbeitsforschung" befördern, indem sie deren Praxisrelevanz durch die Identifizierung von Forschungsbedarfen aus Arbeitnehmerperspektive zu steigern versucht und einen verbesserten Transfer von Forschungsergebnissen in die betrieblichen Anwendungsfelder forciert. Die jüngst begonnene Kooperation zwischen ver.di und dem Fraunhofer Institut für Arbeitswirtschaft und Organisation als fraglos bedeutendem Akteur der Dienstleistungsforschung markiert in diesem Zusammenhang eine wichtige Akzentsetzung. ${ }^{15}$

Neue Chancen für den notwendigen Dialog von Wissenschaft und Gewerkschaften eröffnen sich auch im Blick auf die begonnene Etablierung einer Service Science - einer Dienstleistungswissenschaft als Bedingung der Entwicklung von neuen innovativen Dienstleistungen in verschiedenen Feldern. In diesen können die Gewerkschaften und besonders ver.di die Erfahrungen der Beschäftigten wie auch der Kunden, der Verbraucher usw. einbringen.
Dabei wären unterschiedliche Interessen und Perspektiven zu klären und auszuloten. So kann durch Informationstechnologie gestützte Arbeit im Krankenhaus aus Sicht der Forschung und Entwicklung, des Krankenhausbetreibers, der Pflegekräfte und - last but not least - der Patienten etwas ganz Unterschiedliches bedeuten. Sich darüber von vorneherein zu informieren, zu diskutieren und in der Umsetzung auch mitzubestimmen, sind dabei wichtige Dimensionen gewerkschaftlicher und betrieblicher Interessensvertretung.

\section{FAZIT}

Kern einer Dienstleistungspolitik der Gewerkschaft ver.di ist die ideelle und materielle Aufwertung der Dienstleistungen und der Dienstleistungsarbeit. Wir wollen erstens deutlich machen, dass Investitionen und Innovationen in soziale und ökologische Dienstleistungen ein qualitatives Wachstum und gesellschaftlichen Fortschritt fördern. Dies steht nicht im Gegensatz zu einer Industriepolitik, welche sich ökologisch neu ausrichtet und durch Investitionen und Innovationen in der Elektromobilität, der Energiepolitik, in neuen Technologien Produktivität steigern und Wachstum ermöglichen kann. Allein der ökologische Umbau der Industrie vermag eine halbe Million Arbeitsplätze in der Industrie und fast doppelt so viele Stellen in ökologischen Dienstleistungen zu schaffen. Eine Politik, die sich wie bisher einseitig auf die Förderung der Industrie konzentriert und Technologieförderung als Industrieförderung versteht, ist jedoch kaum in der Lage, ein produktives und arbeitsplatzschaffendes Zusammenwirken von industrieller und Dienstleistungsinnovation voranzutreiben. Die Entwicklung zur Dienstleistungsökonomie in Deutschland könnte und sollte sich von derjenigen in Großbritannien oder in den USA dadurch positiv abheben, dass sie nicht als Kompensation für eine erodierende industrielle Basis entsteht, sondern komplementär und im engen Zusammenhang mit einer modernen und sich modernisierenden industriellen Produktion. Die hiesige Dienstleistungspolitik sollte dies als Chance begreifen und parteiübergreifend der Erkenntnis Rechnung tragen, dass sich gute Dienstleistungen nicht im Selbstlauf entwickeln, sondern aufgewertet, anerkannt und systematisch gefördert werden müssen.

Zweitens gilt es, in Politik und Öffentlichkeit verstärkt bewusst zu machen, dass Dienstleistungen und insbesondere soziale Dienstleistungen keine banalen und minderwertigen Tätigkeiten sind, sondern voraussetzungsvolle und wichtige Beiträge zu wirtschaftlicher Prosperität, sozialer Innovation, individuellem Wohlergehen und gesellschaftlichem Zusammenhalt. Der Arbeitskampf im Sozial- und Erziehungsdienst im Sommer 2009, die Initiative Gute Arbeit, die Kampagne „Der Deckel muss weg" im Gesundheitswesen, die Initiative Faire Arbeit in den Finanzdienstleistungen und im Handel sind Beispiele für die Auseinandersetzung um eine ideelle wie materielle Aufwertung dieser Tätigkeiten.

Eine Dienstleistungspolitik, wie sie ver.di einfordert und für die wir uns engagieren, verfolgt die Ziele guter Arbeit und eines guten Lebens gleichermaßen. Auf diesem Weg sind noch viele Fragen offen, die wir im Dialog mit der Wissenschaft, in der Gesellschaft und auch mit der Politik erörtern und beantworten wollen.

\footnotetext{
14 Ebd., S. 98.

15 In der entsprechenden Vereinbarung wird von beiden Kooperationspartnern sowohl der unangemessen geringe Stellenwert von Dienstleistungen im Bewusstsein von Wirtschaft, Gesellschaft, Forschung, Politik und Medien moniert als auch die Voraussetzungen skizziert werden, die erforderlich sind für eine stärkere Beachtung der Dienstleistungsarbeit bzw. den Wandel hin zu qualitativ hochwertigen, wirtschaftlich tragfähigen Dienstleistungen, zu menschengerechter, professionalisierter Dienstleistungsarbeit und zu einer verstärkten Orientierung auf die Dienstleistungsnehmer.
} 